\title{
Neurofunctional Effects of Developmental Alcohol Exposure in Alcohol-Preferring and Alcohol-Nonpreferring Rats
}

\author{
Maria Tattoli, M.D., Ph.D., Raffaele Cagiano, Ph.D., Silvana Gaetani, Ph.D., \\ Veronica Ghiglieri, Ph.D., Arcangela Giustino, Ph.D., Giampaolo Mereu, Ph.D., \\ Luigia Trabace, Ph.D., and Vincenzo Cuomo, M.D., Ph.D.
}

\begin{abstract}
The neurofunctional effects of developmental alcohol exposure (3\% v/v solution from day 15 of gestation to day 7 after parturition) have been investigated in Sardinian alcoholpreferring $(s P)$ and alcohol-nonpreferring $(s N P)$ rat lines, selectively bred for opposite alcohol preference and consumption. Alcohol exposure significantly decreased the rate of ultrasonic emission in SP male pups; whereas, it did not affect this indicator of emotional reactivity in sNP animals. Perinatal alcohol intake did not influence either learning of an active avoidance task or hippocampal long-term potentiation in both offspring lines. Significant differences in time spent exploring novel objects were observed between control sP and sNP rats subjected to the novel exploration object test. Alcohol exposed $s P$ rats, but not alcohol exposed sNP rats, apparently lost the capacity to discriminate between the novel and the familiar object, even though this difference is difficult to
\end{abstract}

interpret because of the large differences in the respective responses to the novel objects. Neurochemical experiments have shown that basal levels of dopamine (DA) and homovanillic acid (HVA) were significantly higher in the nucleus accumbens (NAC) of $s P$ rats with respect to $s N P$ animals. Perinatal alcohol did not affect basal DA and HVA concentrations or amphetamine-induced DA increase and $H V A$ decrease in the NAC of either $s P$ or $s N P$ offspring. These results suggest that subtle behavioral alterations induced by developmental exposure to low doses of alcohol, which do not cause malformations and/or overt neurotoxicity, may be associated with genetic factors, although not necessarily those responsible for differences in alcohol preference.

[Neuropsychopharmacology 24:691-705, 2001] (C) 2001 American College of Neuropsychopharmacology. Published by Elseiver Science Inc.
KEY WORDS: Perinatal alcohol; Alcohol-preferring rats; Alcohol-nonpreferring rats; Behavior; Electrophysiology; Neurochemistry

From the Department of Pharmacology and Human Physiology (MT, RC, SG, AG, LT, VC), University of Bari, Bari, Italy; and Department of Experimental Biology (VG, GM), University of Cagliari, Cagliari, Italy.

Address correspondence to: Vincenzo Cuomo, M.D., Ph.D., Department of Pharmacology and Human Physiology, University of Bari, Piazza Giulio Cesare 70124, Bari, Italy.

Received 4 April 2000; revised 7 July 2000; accepted 19 September 2000.

\section{INTRODUCTION}

Heavy alcohol intake during pregnancy has been consistently associated with the fetal alcohol syndrome (FAS) in offspring. This syndrome is characterized by growth retardation, morphological abnormalities, and central nervous system (CNS) dysfunction. CNS dysfunction is expressed as a reduced capacity for basic adaptive functioning, including hyperactivity along with attention, learning, and memory deficits (Abel and Hannigan 1995; Streissguth 1986). Because of the heterogeneity of human alcohol consumption and to potential confounding variables surrounding research with human subjects, animal models have become increas- 
ingly important in the analysis of determining factors (i.e., doses, duration and time of exposure, genetic factors) of FAS and alcohol-related neurodevelopmental disorders (ARNDs).

A critical question is to identify risk factors that make some women or their fetuses more susceptible than others to the effects of equivalent amounts of prenatal alcohol exposure (Melcer et al. 1995). It has been hypothesized that genetic factors could contribute to this variability in outcomes observed after gestational alcohol intake (Gilliam and Irtenkauf 1990; Hannigan 1996; Riley and Lochry 1982).

Animal models may provide a valuable tool for assessing gene-based hypotheses of sensitivity to alcohol teratogenesis. To date, an increasing body of evidence indicates that particular genotypes related to alcohol preference, tolerance, or sensitivity may confer specific susceptibility to prenatal alcohol-induced neurofunctional deficits. For example, long sleep mice, bred for extreme loss of the righting reflex after alcohol exposure, produced litters that exhibited poorer learning attributable to prenatal alcohol administration than did relatively alcohol insensitive short sleep mice (Gilliam et al. 1987). Young weanling preferring rats, which differ from nonpreferring ones in preference, tolerance, and sensitivity to alcohol, showed greater sensitivity to teratogenic effects of early neonatal alcohol exposure on locomotor activity than did nonpreferring rats (Riley et al. 1993). Moreover, differential CNS alterations of neonatal alcohol exposure have been found when comparing Maudsley Reactive (MR) and M520 rats; specifically, MR rats displayed greater reduction in cerebellar weight with respect to M520 animals (Goodlett et al. 1989). The aim of the present experiments was to assess whether the neurofunctional effects of developmental alcohol exposure vary in rat lines with different alcohol preferences.

Sardinian alcohol-preferring (sP) and Sardinian alcohol-nonpreferring (sNP) rats have been selectively bred (from Wistar rats) in the Bernard B. Brodie Department of Neurosciences, University of Cagliari, Italy for opposite alcohol preference and consumption. The daily alcohol intake of sP rats is over $4 \mathrm{~g} / \mathrm{kg}$, and the preference ratio for alcohol to water reaches $80-100 \%$; whereas, sNP rats avoid drinking alcohol solution (Colombo et al. 1995; Colombo 1997).

Specifically, the aim of the present study was to investigate, in $\mathrm{sP}$ and sNP male offspring, neurobehavioral, electrophysiological, and neurochemical effects of developmental exposure to alcohol at doses not associated with tolerance, dependence, withdrawal symptoms, or overt signs of toxicity (Battaini et al. 1998; Cagiano et al. 1998; Govoni et al. 1994).

The following neurobehavioral endpoints were evaluated during early postnatal life: (1) ontogeny of motor activity (open field), (2) emotional reactivity (ultrasonic vocalization); and during adulthood: (1) learning (active avoidance task), (2) exploratory behavior, habituation, and visual discrimination (novel exploration object test).

Electrophysiological experiments were carried out in adult offspring in order to determine the influence of perinatal alcohol exposure on in vivo hippocampal long-term potentiation (LTP), the most intensively studied cellular and molecular model for learning and memory (Bliss and Collingridge 1993).

Finally, because alcohol has been shown to affect the mesocorticolimbic dopaminergic system (see Spanagel and Weiss 1999, for references), neurochemical experiments (in vivo microdialysis) were also performed to investigate the effects of developmental exposure to this drug of abuse on extracellular concentrations of dopamine (DA) and homovanillic acid (HVA) as well as on the biochemical responsiveness to a challenge dose of amphetamine in the nucleus accumbens (NAC) of adult offspring.

\section{MATERIALS AND METHODS}

\section{Animals and Treatment Schedule}

The experiments have been conducted in accordance with guidelines released by Italian Ministry of Health (D.L. 116/92), the Declaration of Helsinki, and the Guide for the Care and Use of Laboratory Animals as adopted and promulgated by the National Institutes of Health (USA).

Primiparous alcohol-naive sP and sNP female rats from the 42nd generation of both lines (Department of Neuroscience, Bernard B. Brodie, University of Cagliari, Italy) weighing 280-350 g were used. A randomly bred control line was not established in this selection experiment; furthermore, it was deemed that the use of animals from the original colony (Wistar from Charles River, Italy) at such a long time (about 20 years) from the start of the selection could not provide appropriate control data.

The animals were individually housed in standard plastic cages and allowed free access to food and water, with constant room temperature $\left(20-22^{\circ} \mathrm{C}\right)$ under a reversed 12/12 h light/dark cycle (light on: 21:00 h-09:00 h). Each female was placed with a male rat in the early morning and vaginal smears were taken daily at 19:00 $\mathrm{h}$. The day on which sperm were present was designated day 0 of gestation. To avoid the systemic administration of alcohol, the treatment schedule had to be established on the basis of preliminary studies aimed at assessing the intake of alcohol solution by pregnant sNP females and its temporal distribution. The least biased procedure seemed to be that of controlling both the alcohol solution intake by sP females and the control solution intake by sP and sNP female controls (see 
below) on the basis of the ad libitum alcohol intake by sNP females.

\section{Preliminary Studies}

sNP female rats could consume ad libitum a $3 \%(\mathrm{v} / \mathrm{v})$ aqueous solution of alcohol, from day 15 of gestation to day 7 after parturition, as the only available beverage. Daily alcohol $(\mathrm{g} / \mathrm{kg})$ and total fluid $(\mathrm{ml} / \mathrm{kg})$ intake were measured. One-way analysis of variance (ANOVA) for repeated measures showed that during the exposure period both alcohol $(\mathrm{g} / \mathrm{kg})$ and total fluid $(\mathrm{ml} / \mathrm{kg})$ intake varied significantly $(\mathrm{F}=19.52, \mathrm{df}=12 / 60, p<$ $.001 ; \mathrm{F}=19.80, \mathrm{df}=12 / 60, p<.001)$. A significant increase in alcohol solution intake was observed during the lactation period (Figure 1A, B).

The results showed that about $90 \%$ of alcohol solution intake occurred during the dark phase of the light/ dark cycle. Alcohol solution intake was uniformly distributed during the dark phase. No significant differences in the total alcohol intake between the first and the second half of the dark period were observed (data not shown).

The preliminary studies also showed that the total daily volume of alcohol solution consumed by pregnant sNP rats did not significantly differ from that of sNP animals receiving tap water ad libitum (Figure 1C). This indicated that controlling fluid intake in three of the four experimental groups (see the treatment schedule described below) on the basis of the ad libitum intake of alcohol solution by sNP females was unlikely to produce harmful effects attributable to fluid restriction.

The $3 \% \mathrm{v} / \mathrm{v}$ alcohol concentration was chosen on the basis of our pilot studies showing that the developmental exposure to a higher alcohol concentration $(6 \% \mathrm{v} / \mathrm{v})$ significantly affected such reproduction parameters as
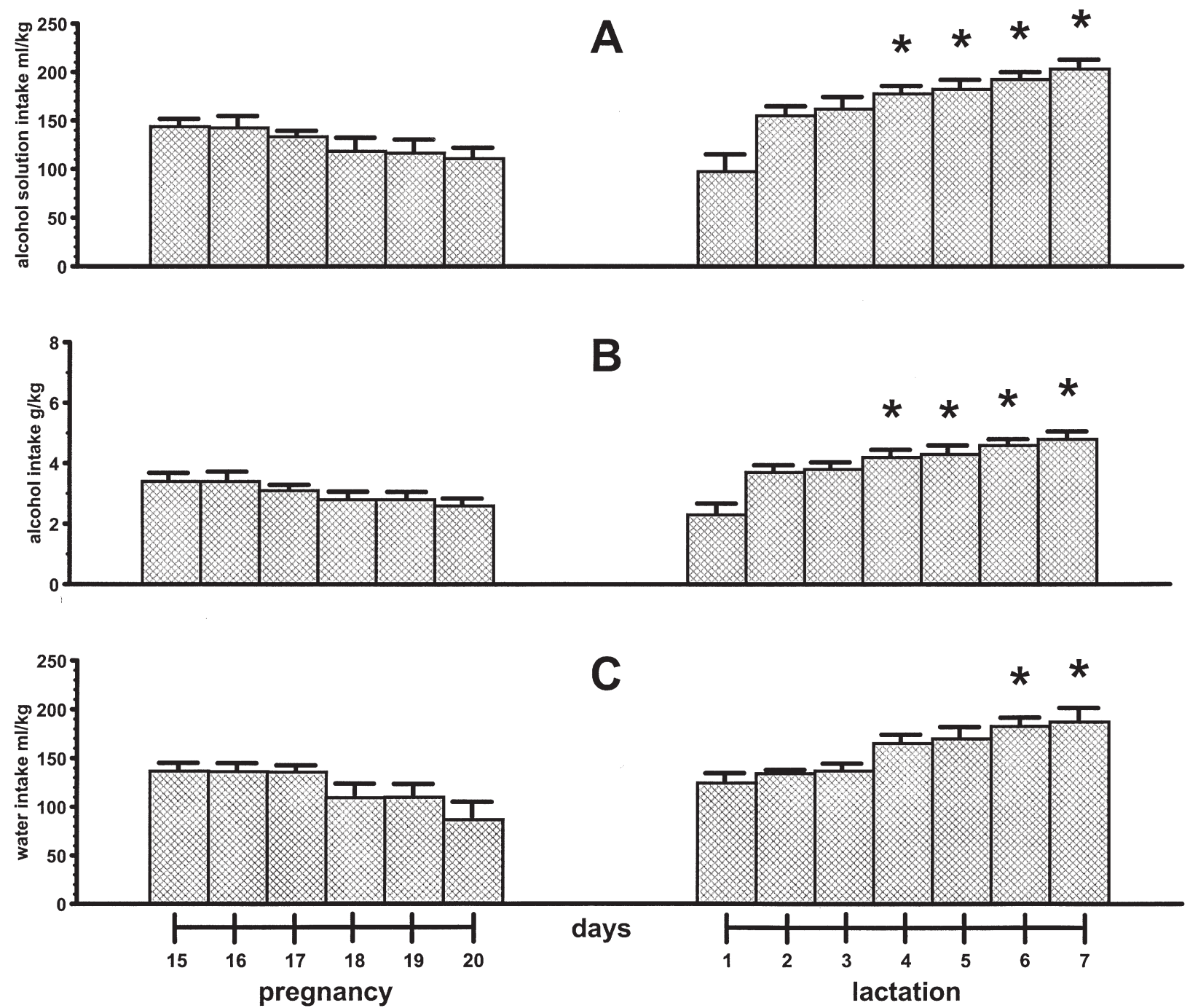

Figure 1. Daily $3 \%(\mathrm{v} / \mathrm{v})$ alcohol solution (A and B) and tap water (C) intake in sNP dams during pregnancy (from day 15 to day 20) and lactation (from day 1 to day 7 after delivery). Each column represents the mean \pm SEM. ${ }^{*} p<.05$ vs. day 15 of gestation (two-tail Dunnett's test). 
dam and pup weight gain as well as litter size at birth. Moreover, the total daily volume of alcohol solution $(6 \% \mathrm{v} / \mathrm{v})$ consumed by pregnant sNP rats was lower than that of sNP animals receiving tap water ad libitum. These results suggested that, at this alcohol concentration, the possible neurofunctional alterations in offspring could be attributable to fluid restriction.

\section{Experimental Procedure}

The exposure schedule used in the present study was as follows.

1. sNP females were given ad libitum a $3 \%(\mathrm{v} / \mathrm{v})$ aqueous solution of alcohol, from day 15 of gestation to day 7 after parturition, as the only available fluid intake (sNP-A).

2. sP females were given daily, from day 15 of gestation to day 7 after parturition, the same volume of aqueous solution of alcohol $(3 \% \mathrm{v} / \mathrm{v})$ consumed by the sNP group (sP-A). To mimic the daily drinking pattern shown by sNP females, sP dams were offered half of the volume at the beginning of the dark period and the remaining volume $6 \mathrm{~h}$ later.

3. Control sNP and sP dams received, as the only available fluid (from day 15 of gestation up to day 7 after parturition), the same volume of a solution in which alcohol was substituted by an equicaloric amount of sucrose (sNP-S and sP-S, respectively). Half of the volume of the solution was given at the beginning of the dark period, and the remaining volume was administered $6 \mathrm{~h}$ later.

This exposure schedule resulted in a daily consumption of alcohol by $\mathrm{sP}$ dams equivalent to that exhibited by sNP ones. Moreover, daily total fluid intake in sucrose-exposed sNP and sP dams was equivalent to that observed in alcohol-exposed sNP and $\mathrm{sP}$ females. There were no differences in food consumption either between lines or between alcohol and sucrose solution groups.

All litters were reduced to a standard size of six male pups per litter (when possible) within $24 \mathrm{~h}$ after birth. All pups were weaned at 21 days of age. One male pup per litter from different litters per treatment group was used in all experiments.

\section{Blood Alcohol Levels (BALs)}

In a series of separate experiments, subgroups of alcohol-exposed sNP and sP dams (eight females for each group) were implanted under anesthesia (Equithesin 3 $\mathrm{ml} / \mathrm{kg} \mathrm{IP}$ ) with catheters in the abdominal aorta on day 20 of gestation, on day 7 after parturition, and $24 \mathrm{~h}$ after alcohol withdrawal. Maternal BALs were measured by a spectrophotometric method (UV-method, Boehringer Mannheim Kit, detection limit $0.22 \mathrm{mmol} / \mathrm{l})$. Drawing time was randomly chosen for each rat during the last 6 hours of the dark phase of the daily dark/light cycle. Blood alcohol determinations were also made in fetuses on day 20 of gestation. All fetuses obtained via hysterotomy were immediately decapitated and blood collected from the neck vessels. Blood samples from fetuses from the same litter were pooled. Twenty-four hours after maternal alcohol withdrawal, pups were decapitated, and blood of each pup was collected from the neck vessels.

Statistical Analysis. Maternal BALs were analyzed by an over-all two-way analysis of variance (ANOVA), followed by a post-hoc test (Tukey's test). Fetal and pup BALs were analyzed by Student's $t$-test.

\section{Reproduction Data}

The body weights of dams (10 females for each group) were taken on days 0 and 20 of gestation. The number of dams giving birth as well as the length of pregnancy were determined. Litter size at birth and postnatal mortality (number of male pups that died before weaning) were evaluated. The body weights of male rats (one pup per litter from 10 different litters per treatment group) were taken.

Statistical Analysis. The general reproduction data were analyzed by over-all two-way or three-way ANOVAs followed by post-hoc tests (Tukey's test) for individual comparisons between groups. Fisher's exact-test was used where appropriate.

\section{Behavioral Studies}

Tests were chosen with the aim to assess various components of the behavioral repertoire with different developmental patterns, thereby minimizing the risk of false-negatives as well as the risk of biases in the interpretation of positive results. At one extreme, for example, ultrasonic calls are produced just hours after birth (Sales 1979; Dastur et al. 1999), which indicates that the neural substrates have completed their development prenatally. At the opposite extreme, active avoidance does not appear before the end of the second postnatal week (Cuomo et al. 1996). In between these two extremes, some responses (particularly motor activity) are given already at birth, but their pattern shows substantial quantitative and qualitative changes over the four postnatal weeks (Laviola et al. 1988).

\section{Ultrasonic Vocalization in Rat Pups}

Recording sessions were conducted in a sound-attenuating chamber $(3.00 \times 2.00 \times 2.00 \mathrm{~m})$ according to the technique previously described by Cagiano et al.(1988). Vocalizations of rat pups were recorded on a Racal 
Store 4DS high-speed tape recorder using a direct mode recording procedure with a tape speed of 30 i.p.s. (76.8 $\mathrm{cm} / \mathrm{s}$ ); the frequency response range was flat between $200 \mathrm{~Hz}$ and $150 \mathrm{kHz}$. Ampex magnetic tapes (length: 3,280 feet, width: 0.25 inch) with precision reels were used. The transducers employed were a calibrated Bruel \& Kjaer (model 4135$) 1 / 4$ in $(0.64 \mathrm{~cm})$ free-field condenser microphone (frequency response flat within \pm 3 $\mathrm{dB}$, from $4 \mathrm{~Hz}$ to $100 \mathrm{kHz}$ ), a Bruel \& Kjaer microphone preamplifier (model 2618) with a linear frequency response from $10 \mathrm{~Hz}$ to $200 \mathrm{kHz}$, which provided $20 \mathrm{~dB}$ amplification, a Rank Precision Ind. low-noise amplifier, which provided $20 \mathrm{~dB}$ amplification steps and a KrohnHite tunable band-pass filter (model 3500) set at 20 to $100 \mathrm{kHz}$

The rate of vocalization (number of calls/15 s) was counted manually by listening to the audible output of the tape recorder with a loudspeaker and by watching the vocalization displayed on Bruel \& Kjaer (model 2033) high- resolution signal analyzer on time-intensity mode during tape replay at $33 / 4$ i.p.s.

One of the six male pups in each litter was randomly removed from the nest (one pup per litter from eight different litters) and placed in a shallow glass dish (15 $\mathrm{cm}$ in diameter and $10 \mathrm{~cm}$ deep) $15 \mathrm{~s}$ before the test. This confined the pup relative to the microphone, which was supported vertically $15 \mathrm{~cm}$ above the dish and thus avoided handling it during the recording session, which lasted $15 \mathrm{~s}$. Ultrasonic vocalization (15 s/ session) was evaluated at the 10th day of age. Each group consisted of eight pups.

Statistical analysis. The statistical analysis of data was based on an over-all two-way ANOVA. Post-hoc tests (Tukey's test) were used for individual between groups comparisons.

\section{Locomotor Activity}

The technique was previously described by Laviola et al. (1988). Briefly, the apparatus was an open field arena $(60 \times 60 \mathrm{~cm})$ made of black Plexiglas, with a light green bottom subdivided by black lines into $12 \times 12 \mathrm{~cm}$ squares. Crossings of square limits with both forepaws were recorded by a counter mechanically activated by the experimenter. All tests were carried out in a soundattenuating cabin $(3 \times 2 \times 2 \mathrm{~m})$ illuminated by a $20-\mathrm{W}$ white light suspended $2 \mathrm{~m}$ above the apparatus. Background noise of $42 \mathrm{~dB}$ was produced by a fan. Animals were subjected to a $30-$ min session at 16 and 23 days of age. The test started by placing the animal at the center of the arena. Immediately after each test, the apparatus was thoroughly cleaned by cotton pads wetted with $96 \%$ ethanol solution. No recourse was made to simultaneous scoring by two experimenters nor to blind procedures, because no experimenter biases were found in previous control experiments in the case of the response considered.

Each experimental group consisted of the following number of animals: 16 days of age (sP-S: $n=9$; sP-A: $n=8$; sNP-S: $n=10$; sNP-A: $n=13$ ); 23 days of age (sPS: $n=10$; sP-A: $n=7$; sNP-S: $n=10$; sNP-A: $n=10$ ).

Statistical Analysis. Data were analyzed by an overall three-way ANOVA for repeated measures for each age. Post-hoc comparisons were made by Dunnett's test.

\section{Novel Exploration Object Test}

The novel exploration object test used in the present study was a modified version (Giustino et al. 1999) of that previously described by Ennaceur and Delacour (1988). Briefly, 90-day-old male rats $(n=10$ for each experimental group) were submitted to two habituation sessions (intersession interval: $24 \mathrm{~h}$ ) where they were allowed $5 \mathrm{~min}$ to explore the apparatus (circular arena, 75-cm diameter). Twenty-four hours after the last habituation session, each rat was placed in the arena and given two 3-min trials with a 1-min intertrial interval. In the first trial (T1) rats were exposed to two identical objects (black and white striped plastic bottles), which constituted samples $A_{1}$ and $A_{2}$. During the second trial (T2) rats were exposed again to two objects, of which one sample was familiar and the other, a new object, B.

Object exploration was quantified as: (1) exploratory activity: total time spent exploring both objects during each trial (T1 and T2); (2) index of global habituation: it is measured by comparing the total time spent exploring the two objects in T1 to that spent in T2; (3) discrimination between the new and the familiar objects: it is measured in $\mathrm{T} 2$ by comparing the time spent exploring the familiar to that spent exploring the new object.

Statistical Analysis. Data were evaluated by using nonparametric statistics because of a highly significant heteroscedasticity in all instances. Statistical analysis was based on Kruskal-Wallis ANOVA followed by post-hoc tests (Wilcoxon paired signed-rank test for within-group comparisons and Wilcoxon-Mann Whitney $U$ test for between-group comparisons). Bonferroni's correction was applied to take into account the increase of Type 1 error probability attributable to the multiple comparison tests performed.

\section{Active Avoidance Conditioning}

The apparatus and procedures have been described previously (De Salvia et al. 1995). Briefly, the apparatus consisted of a two-way avoidance box housed inside a sound-attenuating chamber (Amplifon G-type cabin). Each avoidance box was divided into two compartments connected by an opening of $9 \times 12 \mathrm{~cm}$ and operated by electromechanical programming equipment. 
The conditioned stimulus (CS) consisted of a light (3-W lamp) that was alternately switched on in each compartment. Onset of the CS was followed $12 \mathrm{~s}$ later by the unconditioned stimulus (US), which was a $0.8 \mathrm{~mA}$ scrambled foot shock. The CS remained on during the presentation of the US, which lasted a maximum of $18 \mathrm{~s}$, when both the CS and US were turned off.

A conditioned avoidance response (CAR) was recorded when an animal avoided the US by crossing over to the opposite compartment during the first $12 \mathrm{~s}$ when only the CS was on. An escape response consisted of the animal moving into the opposite compartment following the onset of the US. This response terminated both the CS and the US. Ninety-day-old animals were subjected to a 75 trials session (three 25-trial blocks), with a 60-s intertrial interval (ITI). Each group consisted of 10 animals.

Statistical Analysis. Data were analyzed by a threeway ANOVA for repeated measures followed by a post-hoc test (Dunnett's test).

\section{Electrophysiological Studies}

Animal Preparation. Ninety-day-old animals (sP-S: $n=8$; sP-A: $n=6$; sNP-S: $n=8$; sNP-A: $n=5$ ) were anesthetized with chloral hydrate $(400 \mathrm{mg} / \mathrm{kg}$ IP). Then the rat head was restrained in the stereotaxic apparatus (David Kopf Instruments, Tajunga, CA). Thirty minutes later, anesthesia was maintained with the IP infusion of chloral hydrate, at the rate of $100 \mathrm{mg} / \mathrm{kg} / \mathrm{h}$, via a butterfly needle. This protocol allowed a steady-state, deep level anesthesia throughout the recording session, as indicated by the absence of nociceptive responses. Heart rate and rectal temperature were continuously monitored. Body temperature was maintained at $37^{\circ} \mathrm{C}$ by an autoregulated heating pad.

Electrophysiology. The extracellular recording of the evoked field excitatory postsynaptic potentials (EPSPs) was made in stratum radiatum of the right hippocampal subfield of dendritic layer of CA1 cells. The glass recording electrode (resistance 1.5-2.5 M 2 ) was filled with a freshly filtered solution of $1 \%$ pontamine sky blue in $0.5 \mathrm{M}$ sodium acetate and was positioned at AP -5.2, ML 4.0, and DV 2.5-2.8 mm from bregma according to Paxinos and Watson (1986). Stimulation of the Schaffer collateral/commissural afferents to stratum radiatum was accomplished by using a concentric bipolar electrode $(\varnothing 200 \mu \mathrm{m})$ lowered into the right hippocampus. Coordinates were as follows: AP - 2.9; ML 2.5; DV 2.8-3.0 $\mathrm{mm}$ from bregma with the incisor bar set at -3.3 . Stimuli were constituted by square wave pulses of $30 \mu$ s duration, delivered at a frequency of $0.05 \mathrm{~Hz}$. The evoked EPSP slope was measured from steepest linear part of the descending negativity of the evoked response, just after the presynaptic volley. Samples of
EPSP were taken every $5 \mathrm{~min}$, averaging 10 consecutive responses.

Once the EPSP had been optimized by small adjustments of the recording and stimulant electrodes, it was recorded for at least $10 \mathrm{~min}$ with a stimulation intensity of about $100 \mu \mathrm{A}$, to assess the stability of the recording. Subsequently, an input-output (I/O) relationship was constructed by plotting increasing single stimulus intensity $(5,10,20,40,80,160,320$, and 640 $\mu \mathrm{A})$ versus the evoked EPSP. This curve was used to assess synaptic excitability and to set the stimulus intensity to obtain a test EPSP of about $30-40 \%$ of the maximal response, which is referred to as the baseline stimulus current.

After a baseline period of $30 \mathrm{~min}$, high-frequency stimulation (tetanization) was delivered into the Schaffer pathway. Tetanization consisted of a single, continuous stimulation at $100 \mathrm{~Hz}$ for $1.0 \mathrm{~s}$ using the same intensity as for baseline stimulus current. Post-tetanization responses were followed for at least $120 \mathrm{~min}$. Evoked EPSPs were considered potentiated if their slope was $\geqslant 20 \%$ of baseline. The three temporal phases of EPSP changes after tetanization; that is, (1) post-tetanic potentiation (PTP), (2) short-term potentiation (STP), and (3) long-term potentiation (LTP), were distinguished as previously described (Bliss and Collingridge 1993; Schulz and Fitzgibbons 1997).

Synaptic responses were sampled at 5-10 kHz. Acquisition and analysis were performed by a pCLAMP 5.5/Digidata 1200 system (Axon Instruments Inc., Foster City, CA). At the end of each experiment, the recording site was marked by the iontophoretic ejection of pontamine sky blue by passing \pm DC current $(10 \mu \mathrm{A})$ for $10 \mathrm{~min}$ (four times) through the recording electrode. The stimulated area was marked by the electrolytic lesion generated by DC current $(100 \mu \mathrm{A})$ for $5 \mathrm{~min}$ through the stimulant electrode. The animal was then killed with an IV injection of sodium pentobarbital. Consequently, the upper part of the body was perfused (the descending aorta clamped) with $200 \mathrm{ml}$ of $0.9 \%$ saline followed by $10 \%$ buffered formaldehyde, the brain removed and kept for $24 \mathrm{~h}$ in $10 \%$ formaldehyde. The position of the blue dye (a spot of $20-50 \mu \mathrm{m}$ diameter), and of the electrolytic lesion was verified by a light microscope in serial slices, stained with cresyl violet, and counterstained with neutral red. Only rats in which the histological analysis confirmed that electrodes were correctly positioned were considered.

Statistical Analysis. The post-tetanization EPSP slope was normalized with respect to baseline using the standard formula: $y(t)=$ post-EPSP $(t) /$ pre-EPSP $\times 100$.

Values were expressed as means \pm SEM. Data were evaluated by two-way ANOVA for repeated measures followed by Tukey's test or Student's $t$-test, where appropriate. 


\section{Neurochemical Studies}

90-day-old rats were anesthetized with Equithesin (3 $\mathrm{ml} / \mathrm{kg} \mathrm{IP}$ ) and placed on a stereotaxic apparatus (David Kopf Instruments, Tajunga, CA, USA). Microdialysis was performed in the right shell of NAC.

The skull was exposed, and a small hole was drilled. The dura mater was exposed and removed before inserting the probe at the level of the right shell of the NAC. A vertical dialysis probe $(2 \mathrm{~mm})$ was made of copolymer of acrylonitrile sodium metallylsulfonate (AN69 Hospal SpA; 20,000 Daltons cutoff). Stereotaxic coordinates were as follows: $\mathrm{AP}=+11.8, \mathrm{~L}=+1.2$, $\mathrm{H}=+1.8$ from the interaural line with the incisor bar set at -3.3 according to Paxinos and Watson (1986).

After probe implant, the skin was sutured, and the rats were allowed to recover from anesthesia for at least $15 \mathrm{~h}$ before neurotransmitter release study.

Experiments were performed on freely moving rats. On the day of the experiment, fibers were perfused with Ringer solution containing (mM): $\mathrm{NaCl} 145, \mathrm{KCl} 3$, $\mathrm{CaCl}_{2} 2.2, \mathrm{MgCl}_{2} 1$ in distilled water. The solution was buffered at $\mathrm{pH} 7.4$ with a $2 \mathrm{mM}$ sodium phosphate buffer, filtered $(0.22 \mu \mathrm{m})$ and degassed. Fibers were perfused at a constant flow rate of $2 \mu \mathrm{l} / \mathrm{min}$ with a CMA/100 microinjection pump (Carnegie Medicine, Stockholm, Sweden). After a 60- min wash-out period, consecutive 20-min samples of perfusate were collected and injected into a high-performance liquid chromatography (HPLC) system equipped with an electrochemical detector (ESA, Coulochem II, Bedford, MA, USA) in order to quantitate DA and HVA, according to the technique described by Pozzi et al. (1995). The first electrode was set at $+300 \mathrm{mV}$ (oxidation) and the second at $-225 \mathrm{mV}$ (reduction). Separation was obtained by us- ing a reverse phase column (LC-18 DB, $15 \mathrm{~cm}, 5 \mu \mathrm{m}$ particle size, Supelco). The mobile phase, consisting of $13.6 \mathrm{~g} / \mathrm{l}$ of sodium acetate, $37 \mathrm{mg} / \mathrm{l}$ of disodium EDTA dihydrate, $80 \mathrm{mg} / 1$ of octyl sodium sulfate, $60 \mathrm{ml} / 1$ of methanol, pH 4.1 with acetic acid, was pumped at a constant flow rate of $1.0 \mathrm{ml} / \mathrm{min}$ (Shimadzu LC-10AD).

Once a stable basal DA and HVA output were obtained (no more than 10\% difference between three consecutive samples) rats were given a challenge dose of amphetamine $(0.5 \mathrm{mg} / \mathrm{kg})$. d-Amphetamine sulfate (Sigma Chemical Company, St. Louis, MO, USA) was dissolved in saline and administered (as the salt) SC in a volume of $2 \mathrm{ml} / \mathrm{kg}$.

The position of the microdialysis probe was verified by histological procedures at the end of each experiment (Figure 9). Only in rats where probe tracks were exactly located in the target area were considered in the results.

Each experimental group consisted of the following number of animals: sNP-S $=8$; sNP-A $=7$; sP-S $=9$; $\mathrm{sP}-\mathrm{A}=8$.

Statistical Analysis. Data were analyzed by a threeway ANOVA for repeated measures followed by a post-hoc test (Dunnett's test).

\section{RESULTS}

\section{Blood Alcohol Levels (BALs)}

As shown in Figure 2, the exposure to a 3\% (v/v) alcohol solution, from day 15 of pregnancy to day 7 after delivery, resulted in equivalent BALs in $\mathrm{sP}$ and $\mathrm{sNP}$ dams. BALs were significantly increased on day 7 after delivery with respect to those found on day 20 of preg-

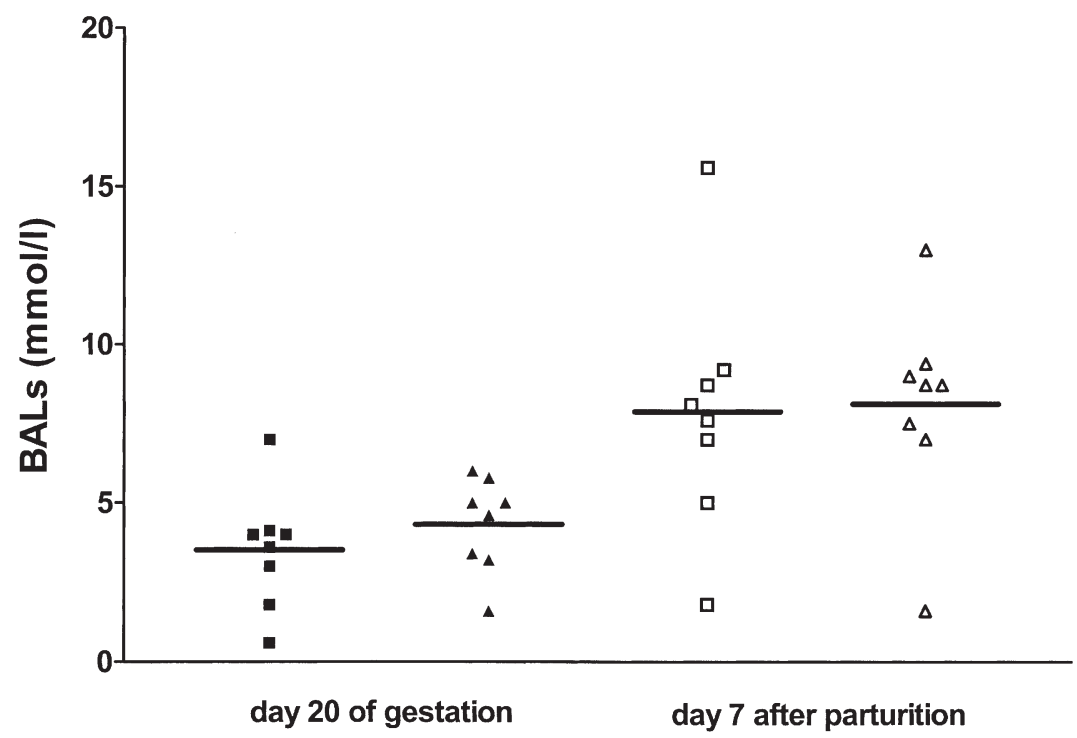

Figure 2. Blood alcohol levels (BALs mmol/l) of sP (triangles) and sNP (squares) dams on day 20 of gestation and on day 7 after delivery. Each point represents an individual observation. Horizontal lines indicate the mean. 
nancy in both animal lines $\left(\mathrm{F}_{\text {rat lines }}=0.32, \mathrm{df}=1 / 28, \mathrm{~ns}\right.$; $\mathrm{F}_{\text {times }}=17.38, \mathrm{df}=1 / 28, p<.0005 ; \mathrm{F}_{\text {rat lines } \times \text { times }}=.06$, $\mathrm{df}=1 / 28, \mathrm{~ns})$. Twenty-four hours after the end of perinatal exposure, alcohol was undetectable in the blood of both sP and sNP dams.

Fetal BALs (mean mmol/1 \pm SEM) on day 20 of gestation were as follows: $\mathrm{sP}=3.6 \pm 0.4 ; \mathrm{sNP}=2.8 \pm 0.4$. The statistical analysis of fetal BALs (Student's $t$-test) showed no significant differences between the two lines. Twentyfour hours after maternal alcohol withdrawal, alcohol was undetectable in the blood of both sP and sNP pups.

\section{Reproduction Data}

Over-all two-way ANOVAs showed that dam weight gain, pregnancy length, and litter size at birth were not significantly affected by perinatal exposure to alcohol in both $\mathrm{sP}$ and $\mathrm{sNP}$ rat lines. Over-all three-way ANOVA for repeated measures showed that perinatal alcohol exposure did not affect male pup weight gain. Moreover, Fisher's exact test revealed that alcohol exposure did not influence postnatal mortality of male pups in both lines of rats (data not shown).

\section{Behavioral Studies}

Ultrasonic Vocalization in Rat Pups. An over-all two-way ANOVA for ultrasonic calls gave the following differences: $\mathrm{F}_{\text {rat lines }}=0.79, \mathrm{df}=1 / 28, \mathrm{~ns} ; \mathrm{F}_{\text {treatments }}=$ $4.76, \mathrm{df}=1 / 28, p<.05 ; \mathrm{F}_{\text {rat lines }} \times$ treatments $=5.36, \mathrm{df}=$ $1 / 28, p<.05$. Individual comparisons (Tukey's test) revealed that perinatal exposure to alcohol significantly decreased the number of ultrasounds $/ 15 \mathrm{~s}$ in $\mathrm{sP}$ rats; whereas, no significant changes were observed in sNP animals (Figure 3 ).
Locomotor Activity. Three-way repeated measures ANOVAs for open field crossings made during a 30min session showed that perinatal treatment with alcohol did not significantly affect locomotor activity either in 16- or 23-day- old sP and sNP offspring (data not shown).

Novel Exploration Object Test. Kruskal-Wallis ANOVA for total exploratory activity during T1 showed the following significant difference: $\mathrm{H}=25.04, \mathrm{df}=3, p<$ .001. Between-group comparisons showed that the time spent exploring the objects during T1 was significantly lower in 90-day-old sP-S rats with respect to SNP-S animals. Perinatal exposure to alcohol did not affect exploratory activity during $\mathrm{T} 1$ in either $\mathrm{sP}$ or $\mathrm{sNP}$ rats. As to global habituation, within-group comparisons showed that sNP-S and sNP-A rats exhibited lower levels of exploratory activity during T2 as compared to those found in T1; whereas, no significant difference in the exploration time between T1 and T2 was found in sP-S and sP-A animals (Figure 4). Kruskal-Wallis ANOVA for discrimination showed the following difference: $\mathrm{H}=12.85, \mathrm{df}=3, p<.01$. Within-group comparisons showed that time spent by both sNP-S and sP-S rats in exploring the familiar object during $\mathrm{T} 2$ was significantly lower than that spent in exploring the novel object. Perinatal exposure to alcohol did not affect discrimination in sNP animals, and no significant difference in the exploration time of the novel with respect to the familiar object was found in $\mathrm{sP}$ rats (Figure 5).

Active Avoidance Conditioning. An over-all threeway repeated measures ANOVA for CARs showed that perinatal exposure to alcohol did not affect the acquisi-

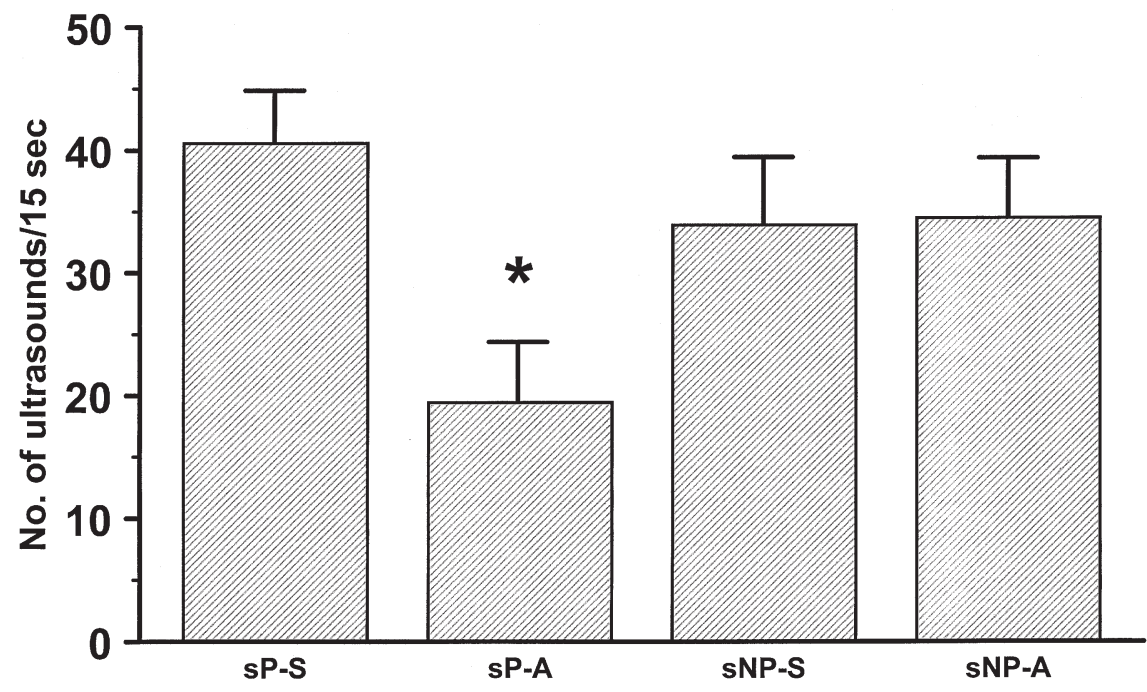

Figure 3. Effects of perinatal alcohol exposure on ultrasonic vocalization rate of 10-day old male pups. Each column is the mean \pm SEM. ${ }^{*} p<.05$ vs. sP-S (Tukey's test). 


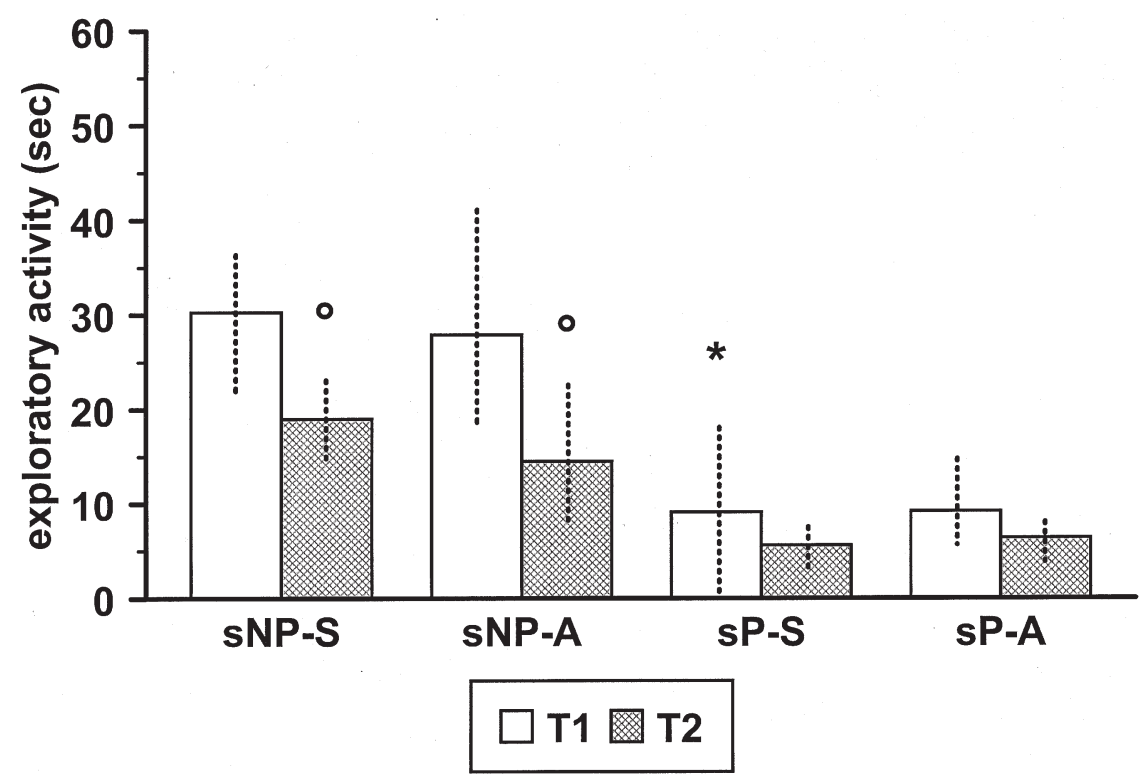

Figure 4. Effects of perinatal exposure to alcohol on exploratory activity during Trial 1 (T1) and Trial 2 (T2) of 90-day-old sP and sNP rats. Data were expressed as median values and interquartiles (dashed line). ${ }^{*} p<.01$ vs. T1 sNP-S (WilcoxonMann Whitney U test with Bonferroni's correction); ${ }^{\circ} p<.005$ vs. T1 of the same group (Wilcoxon Paired Signed-Rank test).

tion of an active avoidance task in 3-month-old sNP and sP rats (Figure 6).

\section{Electrophysiological Studies}

Analysis of I/O relationship indicated that there were no significant differences among groups. Hence, even if no statistically significant changes were found in the baseline excitability of the CA3-CA1 projection across the var- ious groups of animals, at high stimulus intensity $(>200$ $\mu \mathrm{A}) \mathrm{I} / \mathrm{O}$ curves of alcohol exposed sP and sNP groups exhibited a trend toward a higher level with respect to those shown by sucrose-treated groups (Figure 7A).

Similarly, the analysis of post-tetanus behavior of normalized EPSP slopes demonstrated that there were no significant changes in LTP induction and expression across the groups (Figure 7B). Indeed, PTP, STP, and LTP expression were comparable. The potentiation ob-

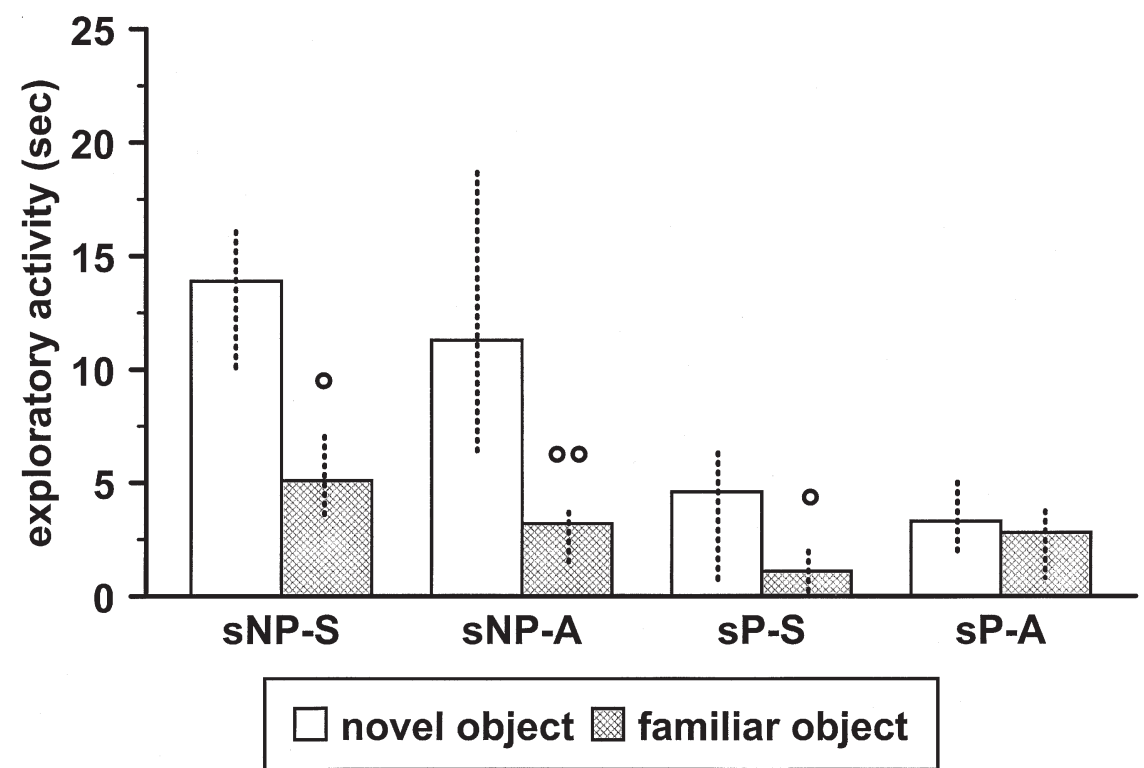

Figure 5. Effects of perinatal exposure to alcohol on exploratory activity during Trial 2 of 90-day-old sP and sNP rats. Data were expressed as median values and interquartiles (dashed line). ${ }^{\circ} p<.05,{ }^{\circ} p<.01$; vs. novel object (Wilcoxon Paired Signed-Rank test). 


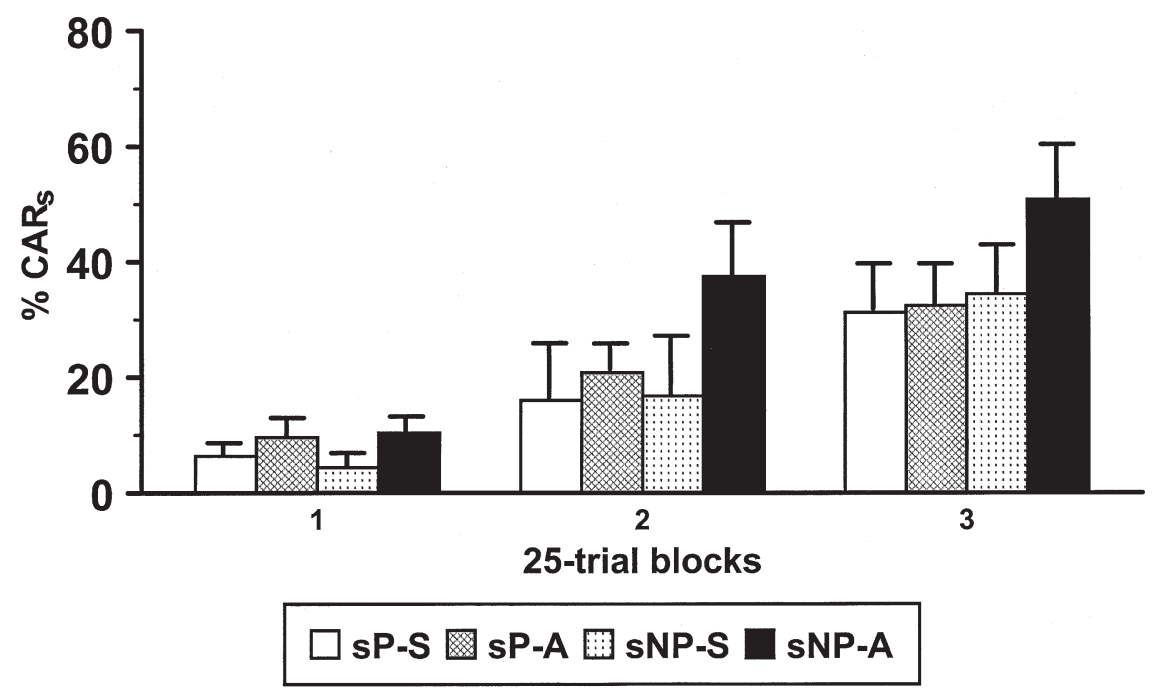

Figure 6. Influence of perinatal exposure to alcohol on active avoidance learning in 90-day old rats. CAR $=$ Conditioned Avoidance Response. Each column is the mean \pm SEM.

served after the tetanization was attributed to an actual increase in synaptic efficacy, because the mean amplitude of the presynaptic volley was not different from control baseline (data not shown).

\section{Neurochemical Studies}

Effects of Amphetamine Challenge on Extracellular DA Concentrations in the Shell of the NAC of Perinatally Sucrose- and Alcohol-Exposed sP and sNP Rats. A three-way ANOVA for repeated measures of basal DA concentrations (two consecutive samples collected before amphetamine challenge) indicated that basal DA concentrations (Figure 8A) were significantly higher in $\mathrm{sP}$ with respect to sNP offspring, both in alcohol- and sucrose-treated animals $\left(\mathrm{F}_{\text {rat lines }}=10.09, \mathrm{df}=1 / 28, p<\right.$ $.005)$.

Because differences between times and between treatments $\times$ times were not significant, post-hoc tests for individual comparisons were not performed.

A three-way ANOVA for repeated measures of changes in extracellular DA concentrations (actual values) elicited by a challenge dose of amphetamine (last basal value and six consecutive samples after amphetamine challenge) gave the following differences: $\mathrm{F}_{\text {rat lines }}=4.26, \mathrm{df}=1 / 28, p<.05 ; \mathrm{F}_{\text {treatments }}=0.06, \mathrm{df}=1 / 28$,
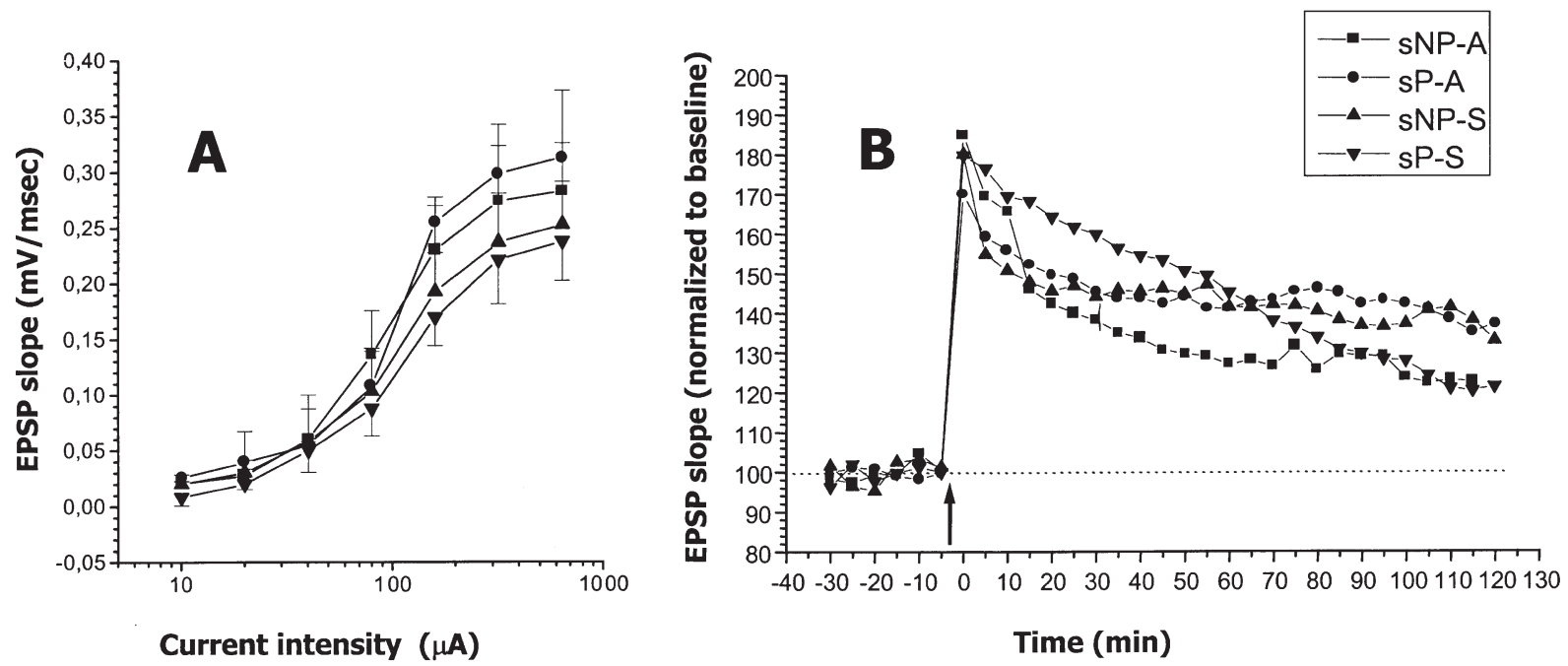

Figure 7. (A) Basal, pretetanus, I/O relationship. The I/O curves were constructed by plotting evoked EPSP-slope vs. stimulus intensity (scan: 5 to $640 \mu \mathrm{A}$ ). Each value of EPSP slope (mean \pm SEM) is the average of 10 consecutive responses taken every $5 \mathrm{~min}$. (B) LTP. Averages (mean) of EPSP slopes were normalized to the pretetanus period. Each point represents the average of 10 consecutive responses taken every 5 min. 

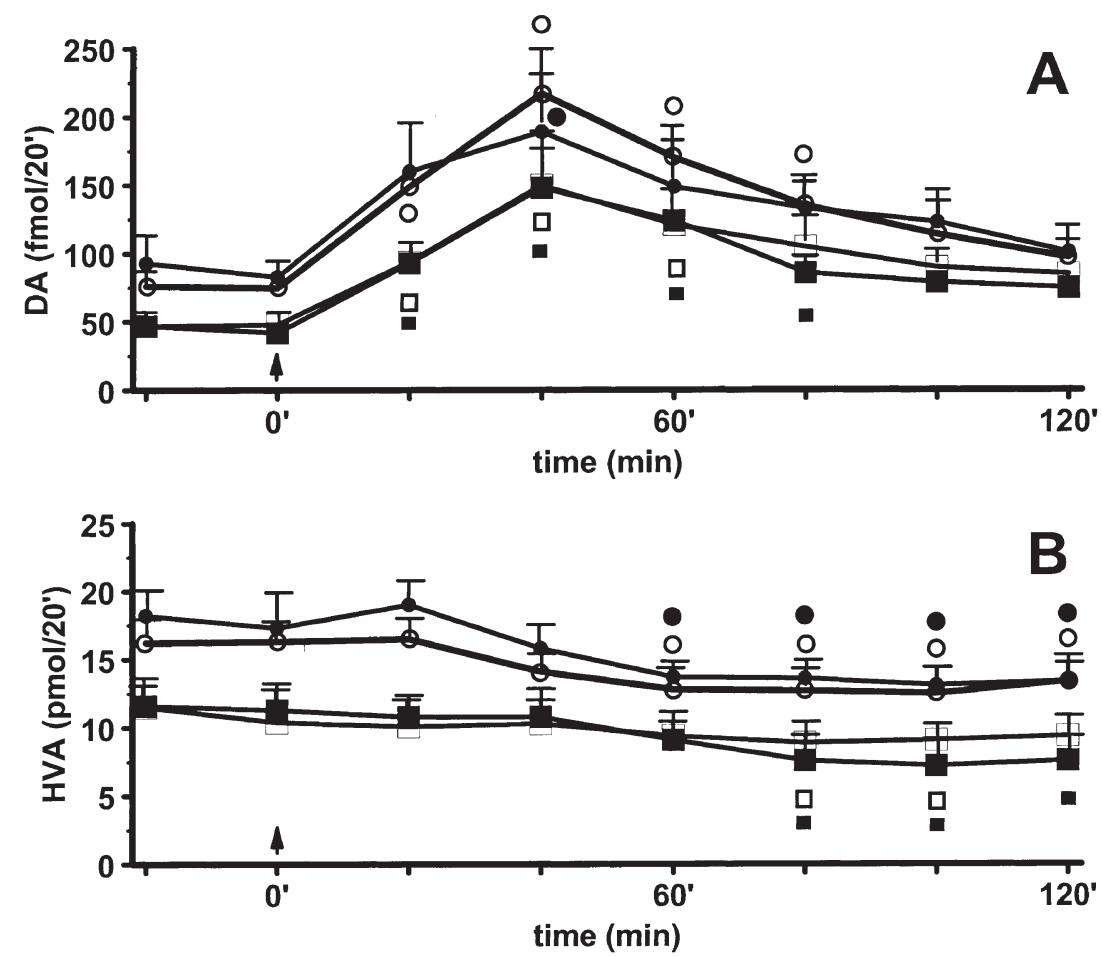

Figure 8. Effects of amphetamine challenge $(0.5 \mathrm{mg} / \mathrm{kg} \mathrm{SC}, \uparrow)$ on extracellular DA (A) and HVA (B) concentrations in the NAC of 90-day-old sP and sNP rats perinatally exposed to sucrose ( $\bigcirc \bigcirc$ sP-S; $\square-\square$ sNP-S) or alcohol ( $-\bigcirc \mathrm{sP}-\mathrm{A}$;

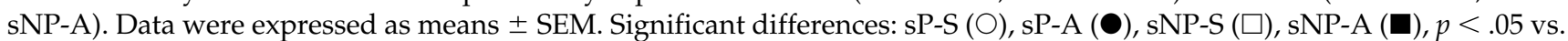
last basal sample (Dunnett's test).

$\mathrm{ns} ; \mathrm{F}_{\text {times }}=30.18, \mathrm{df}=6 / 168, p<.0001 ; \mathrm{F}_{\text {rat lines } \times \text { treatments }}=$ $0.02, \mathrm{df}=1 / 28, \mathrm{~ns} ; \mathrm{F}_{\text {rat lines } \times \text { times }}=0.61, \mathrm{df}=6 / 168, \mathrm{~ns}$; $\mathrm{F}_{\text {treatments }} \times{ }_{\text {times }}=0.30, \mathrm{df}=6 / 168, \mathrm{~ns} ; \mathrm{F}_{\text {rat lines } \times \text { treatments } \times}$ times $=0.53, \mathrm{df}=6 / 168$, ns.

Within-group comparisons (Dunnett's test) showed that amphetamine administration induced a significant increase in extracellular DA concentrations (actual values) with respect to the last basal sample in both alcohol- and sucrose-treated sP and sNP animals.

Effects of Amphetamine Challenge on Extracellular HVA Concentrations in the Shell of the NAC of Perinatally Sucrose- and Alcohol-Exposed sP and sNP Rats. A three-way ANOVA for repeated measures of basal HVA concentrations (two consecutive samples collected before amphetamine challenge) indicated that basal HVA concentrations (Figure 8B) were significantly higher in $\mathrm{SP}$ with respect to $\mathrm{sNP}$ offspring, both in alcohol- and sucrose-treated animals $\left(\mathrm{F}_{\text {rat lines }}=6.29\right.$, $\mathrm{df}=1 / 25, p<.02)$.

Because differences between times and between treatments $\times$ times were not significant, post-hoc tests for individual comparisons were not performed.

A three-way ANOVA for repeated measures of changes in extracellular HVA levels (actual values) elicited by a challenge dose of amphetamine (last basal value and six consecutive samples after amphetamine challenge) gave the following differences: $\mathrm{F}_{\text {rat lines }}=$ $8.13, \mathrm{df}=1 / 28, p<.01 ; \mathrm{F}_{\text {treatments }}=0.16, \mathrm{df}=1 / 28, \mathrm{~ns}$; $\mathrm{F}_{\text {times }}=20.79, \mathrm{df}=6 / 168, p<.0001 ; \mathrm{F}_{\text {rat lines } \times \text { treatments }}=$ $0.12, \mathrm{df}=1 / 28, \mathrm{~ns} ; \mathrm{F}_{\text {rat lines } \times \text { times }}=1.38, \mathrm{df}=6 / 168, \mathrm{~ns}$; $\mathrm{F}_{\text {treatments } \times \text { times }}=1.07, \mathrm{df}=6 / 168, \mathrm{~ns} ; \mathrm{F}_{\text {rat lines }} \times$ treatments $\times$ times $=0.32$, df $=6 / 168$, ns.

Within-group comparisons (Dunnett's test) showed that amphetamine administration induced a significant decrease in extracellular HVA concentrations (actual values) with respect to the last basal sample either in alcohol- or sucrose-treated sP and sNP animals.

\section{DISCUSSION}

The results of the present study indicate that the susceptibility to short- and long-term neurofunctional alterations induced by perinatal exposure to alcohol, at doses below those associated with gross malformations and/or overt neurotoxic effects, can be influenced by genetic factors, although not necessarily those responsible for differences in alcohol preference. An unavoidable bias in this analysis was the lack of an appropriate control group in the absence of a randomly bred control line in the original selection experiment. The differen- 
tial sensitivity of sNP and sP offspring in response to developmental alcohol exposure is endpoint specific.

Lack of significant changes in maternal and pup weight gain suggests that nutritional deficiency was not a factor in the alcohol offspring outcome. Moreover, perinatal alcohol treatment produced equivalent BALs in the sP and sNP lines, eliminating the possibility that differential alcohol effects were produced by differences between lines in the rate of alcohol metabolism. Furthermore, alcohol is no longer detectable in the blood of dams and male pups on day 8 after parturition ( $24 \mathrm{~h}$ after the end of the treatment) and, thus, the alterations observed in the offspring are unlikely to be caused by direct actions of this drug of abuse. This confirms the high vulnerability of the developing brain to harmful effects of alcohol.

The results of the present experiments show that perinatal alcohol exposure significantly decreased the rate of ultrasonic emission in sP pups; whereas, it did not affect this behavioral parameter in SNP animals; actually, this was the only substantial difference in alcohol effects between lines.

These results are in agreement with previous studies showing that maternal alcohol consumption significantly reduced ultrasonic vocalization in rat offspring (Hard et al. 1985; Kehoe and Shoemaker 1991). The decrease in vocalization during the neonatal period is interesting in view of the observation that prenatally alcohol-exposed newborn pups are less able to attract the attention of their mother than are control pups (Abel and Greizerstein 1982). In this regard, another example of altered neonatal behavior resulting in altered dam response is that pups exposed to alcohol prenatally are unable to elicit retrieval from a dam as quickly as control pups (Ness and Franchina 1990).

The reduced rate of calling produced by perinatal alcohol exposure reflects a decrease in the emotional reactivity in sP offspring. Indeed, the rate of calling (number of ultrasonic calls per time unit) is a reliable indicator of the emotional state of the rat. Anxiolytic drugs selectively reduce the rate of calling; whereas, anxiogenic agents increase the number of calls/time unit (Insel et al. 1986). Moreover, ultrasonic vocalization provides a valuable tool for detecting changes in emotional reactivity produced by adverse developmental treatment with a potential for extrapolation to humans. In fact, ultrasonic emissions in rodents have communication purposes, and it has been suggested that this function may be related to human infant crying (Elsner et al. 1990). In this regard, several developmental studies in humans have shown that peculiar changes in acoustic features of neonatal cries seem to be an early indicator of long-term alterations in the neurophysiological status caused by adverse pre- and postnatal events (Lester 1987; Michelsson et al. 1977).

The results obtained in the present study are in agreement with clinical data showing that infants born to mothers who drink alcohol during pregnancy display altered crying and emotional instability (see Nulman et al.1998 for references). These effects may have significant impact on subsequent mother-infant interactions.

Similarly, because alterations in rat pup ultrasonic calling influence maternal behavior, which, in turn, might affect the behavior of the offspring, the changes in ultrasonic vocalization elicited by perinatal exposure to alcohol could have a role in the production of behavioral abnormalities observed in adult sP animals.

The present findings show that perinatal exposure to low doses of alcohol did not affect the acquisition of an active avoidance task in either adult sP or sNP offspring. The lack of changes in learning capacities was paralleled by electrophysiological data indicating that either sP-A or sNP-A rats did not exhibit significant alterations in hippocampal (CA1 region) LTP, which reflects cellular physiological changes critical to the processes of learning and to the intermediate stages of memory consolidation and retention (Bliss and Collingridge 1993).

Our data are in agreement with recent findings showing that prenatal alcohol exposure does not alter LTP in the hippocampus of rat offspring (Krahl et al. 1999). Concerning the novel exploration object test, object recognition, which is assessed from the preference normal rats display for investigating novel rather than familiar objects, provides a valid and relatively pure measure of nonspatial working memory (Ennaceur and Delacour 1988; Giustino et al. 1999). The present results have shown significant differences in time spent exploring novel objects between adult SP-S and sNP-S rats.

The lower exploratory activity of sP-S offspring could be partly attributed to an altered responsiveness to situations requiring adaptation to novel environmental stimuli. Specifically, the neophobia displayed by sP-S rats in the present experiments could be attributed to their greater innate degree of anxiety with respect to sNP ones (Colombo et al. 1995).

Developmental alcohol exposure did not influence exploratory activity in $\mathrm{T} 1$ in both $\mathrm{sP}$ and $\mathrm{sNP}$ rats; whereas, lack of habituation (lower levels of exploratory activity during $\mathrm{T} 2$ compared to those found in T1) was observed in both sP-S and sP-A with respect to sNP-S and sNP-A animals. However, because the basal activity levels were somewhat low in $\mathrm{sP}$ rats, we cannot exclude that the lack of habituation upon the second presentation of the objects for sP-S and sP-A groups could be attributed to a "floor effect" (i.e., exploration was too low to decrease much further).

Both SNP-S and SP-S rats were able to discriminate between the novel and the familiar object. sP-A rats, but not sNP-A rats, apparently lost this capacity to discriminate; this difference, however, is difficult to interpret because of the large differences in the respective responses to the novel object. 
Previous studies (Kim et al. 1997) have shown that developmental alcohol exposure did not affect objectrecognition performance; whereas, it produced deficits in the water maze task. According to these authors, the mechanisms underlying spatial cognitive abilities seem to be more vulnerable to the teratogenic effects of prenatal alcohol exposure than those underlying objectrecognition abilities.

The between-lines differences in alcohol-related injuries observed in the present experiment raise the question whether different stress experiences in rats of the two lines could have influenced the sensitivity to harm- ful alcohol effects. For example, sNP rats forced to drink alcohol may have experienced greater stress than $\mathrm{sP}$ rats, and this may have contributed to the differences in alcohol effects.

Because multiple neurotransmitter systems modulate the behavioral endpoints affected by perinatal alcohol exposure (ultrasonic emission and novel object exploration), experiments are in progress to explore the neurochemical mechanisms underlying these behavioral changes.

As far as DA is concerned, the present results indicate that the basal levels of DA and its major metabo-

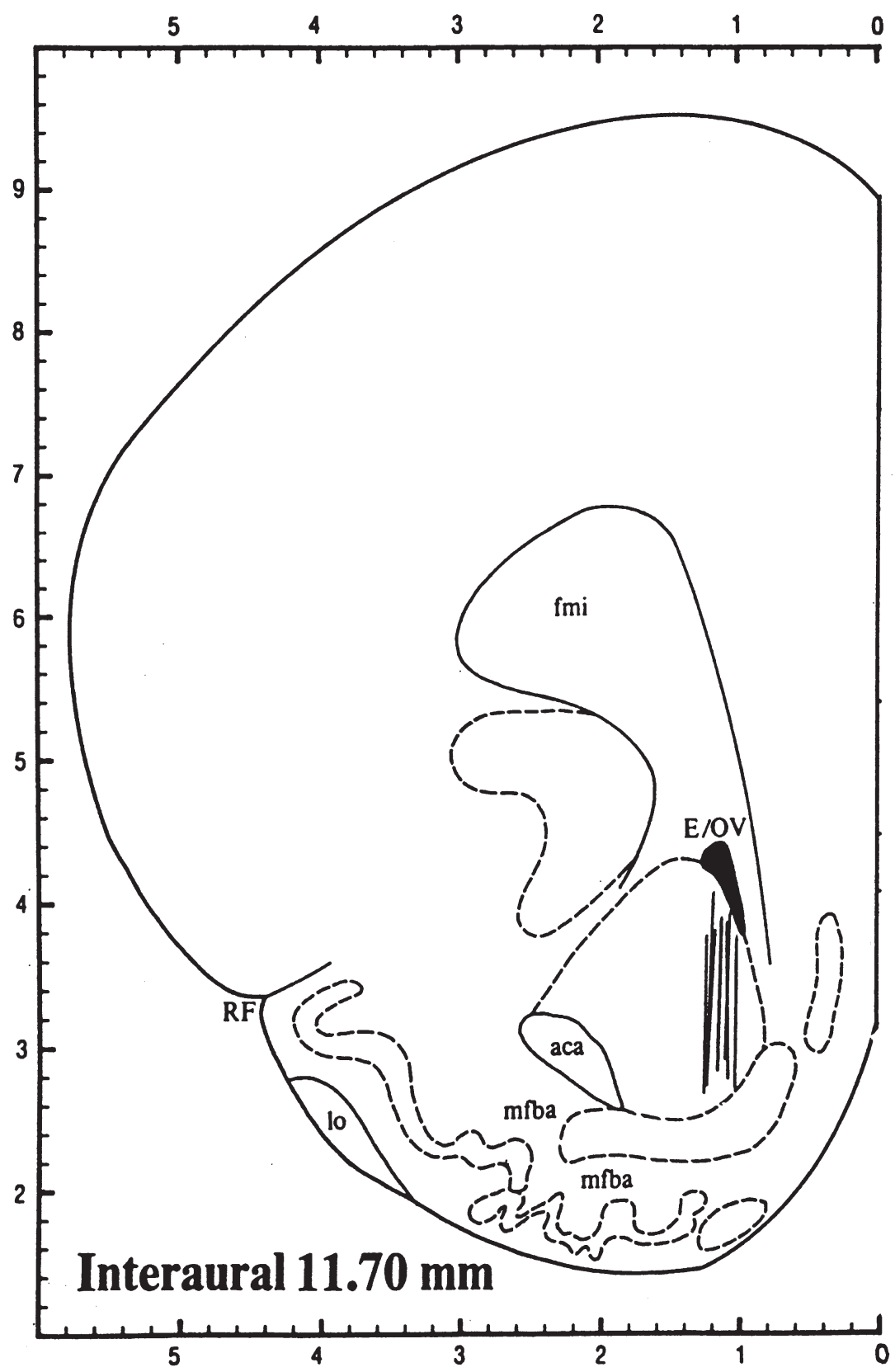

Figure 9. Forebrain section, redrawing from Paxinos and Watson (1986), represents the track corresponding to the dialyzing portion of the probes implanted in the NAC shell. 
lite HVA were significantly higher in the NAC of sP rats as compared with the SNP animals. These differences are present in both alcohol- and sucrose-treated animals.

Previous findings have revealed that differences between rat lines used in the present experiments and other genetically selected for alcohol preference or avoidance (Indiana lines) concern the dopaminergic system. In fact, although Indiana alcohol-preferring rats exhibit lower DA concentrations in the NAC as well as in other forebrain areas when compared with those found in Indiana nonpreferring animals (Murphy et al. 1982), no differences in DA levels have been observed between sP and sNP rats (Devoto et al. 1998).

On the other hand, the Indiana preferring as well as sP lines show reduced forebrain densities of D1 (De Montis et al. 1993) and D2 (McBride et al. 1993; Stefanini et al. 1992) receptors. Our present data, showing higher DA levels in the NAC of sP rats with respect to sNP animals could be consistent with these last findings. Indeed, it could be hypothesized that the increased DA extracellular concentrations observed in $\mathrm{sP}$ rats could down-regulate D1 and D2 receptors. Furthermore, the present experiments have shown that perinatal acohol exposure did not affect either basal DA levels or the amount of temporal pattern of amphetamineinduced increase in DA and decrease in HVA concentrations in the NAC of both rat lines.

In this regard, the majority of studies (see Maier et al. 1996 for references) examining the effects of developmental exposure to alcohol on neurotransmitter levels have furnished results that are divergent (increase, decrease, or no change). In fact, it has been shown that a binge-like alcohol exposure throughout gestation reduced the content of DA in the entire fetal brain (Maier et al. 1996). Moreover, adult rats prenatally exposed to alcohol showed no changes in baseline DA levels and an increased DA release (NAC and striatum) in response to a challenge dose of alcohol (Blanchard et al. 1993). In addition, prenatal alcohol treatment significantly reduced the number of spontaneously active DA neurons in the substantia nigra and ventral tegmental area in 3- and 5-month-old male offspring (Shen et al. 1999). However, the comparison of the present data with those reported in the literature should take into account several factors, including the period of the developmental exposure, alcohol administration paradigm, the dose, the dietary factors, the time of postnatal assessment, and testing procedures.

In sum, the present findings, showing a selective differential sensitivity of sP and sNP lines to developmental low alcohol exposure, suggest that genetic factors may confer specific susceptibility to ARNDs. Furthermore, ad-hoc experiments are needed to assess whether or not these factors are the same as those responsible for differences in alcohol preference.

\section{ACKNOWLEDGMENTS}

This study was supported by a grant from the MURST (Cofinanziamento-97). The authors thank Prof. Giorgio Bignami for his valuable suggestions.

\section{REFERENCES}

Abel EL, Greizerstein HB (1982): Growth and development in animals prenatally exposed to alcohol. In Abel E (ed), Fetal Alcohol Syndrome, vol. III, Animal Studies. Boca Raton, CRC Press, pp 39-57

Abel EL, Hannigan JH (1995): Maternal risk factors in fetal alcohol syndrome: Provocative and permissive influences. Neurotoxicol Teratol 17(4):445-462

Battaini F, Pascale A, Ancona DMR, Cagiano R, Cuomo V, Govoni S (1998): Moderate alcohol intake: Behavioral and neurochemical correlates in rats. Nutr Neurosci 1:151-159

Blanchard BA, Steindorf S, Wang S, LeFevre R, Mankes RF, Glick SD (1993): Prenatal ethanol exposure alters ethanol-induced dopamine release in nucleus accumbens and striatum in male and female rats. Alc: Clin Exp Res 17:974-981

Bliss TVP, Collingridge GL (1993): A synaptic model of memory: Long-term potentiation in the hippocampus. Nature 361:31-39

Cagiano R, Barfield RJ, White NR, Pleim ET, Weinstein M, Cuomo V (1988): Subtle behavioral changes produced in rat pups by in utero exposure to haloperidol. Eur J Pharmacol 157:45-50

Cagiano R, Tattoli M, Persichella M, Mazzoccoli M, Govoni S, Cuomo V (1998): Effects of chronic low dose of ethanol intake on sexual behavior in rats. Alcohol 16(2):1-4

Colombo G, Agabio R, Lobina C, Reali R, Zocchi A, Fadda F, Gessa GL (1995): Sardinian alcohol-preferring rats: A genetic animal model of anxiety. Physiol Behav 57: 1181-1185

Colombo G (1997): Ethanol drinking behavior in Sardinian alcohol-preferring rats. Alc Alcoholism 32:442-454

Cuomo V, De Salvia M, Petruzzi S, Alleva E (1996): Appropriate endpoints for the characterization of behavioral changes in developmental toxicology. Environ Health Persp 104:307-315

Dastur FN, McGregor IS, Brown RE (1999): Dopaminergic modulation of rat pup ultrasonic vocalizations. Eur J Pharmacol 382:53-67

De Montis MG, Gambarana C, Gessa GL, Meloni D, Tagliamonte A, Stefanini E (1993): Reduced [ $\left.{ }^{3} \mathrm{H}\right] \mathrm{SCH} 23390$ binding and DA-sensitive adenyl cyclase in the limbic system of ethanol-preferring rats. Alc Alcoholism 28:397-400

De Salvia MA, Cagiano R, Carratù MR, Di Giovanni V, Trabace L, Cuomo V (1995): Irreversible impairment of active avoidance behavior in rats prenatally exposed to mild concentration of carbon monoxide. Psychopharmacology 122:66-71

Devoto P, Colombo GC, Stefanini E, Gessa GL (1998): Sero- 
tonin is reduced in the frontal cortex of Sardinian ethanol-preferring rats. Alc Alcoholism 33:226-229

Elsner J, Suter D, Alder S (1990): Microanalysis of ultrasound vocalization of young rats: Assessment of the behavioral teratogenicity of methylmercury. Neurotoxicol Teratol 12:7-14

Ennaceur A, Delacour J (1988): A new one-trial test for neurobiological studies of memory in rats. I: Behavioral data. Behav Brain Res 31:47-59

Gilliam DM, Stilman A, Dudek BC, Riley EP (1987): Fetal alcohol effects in long- and short-sleep mice: Activity, passive avoidance, and in utero ethanol levels. Neurotoxicol Teratol 9:349-357

Gilliam D, Irtenkauf K (1990): Maternal genetic effects on ethanol teratogenesis and dominance of relative embryonic resistance to malformations. Alc: Clin Exp Res 14:539-545

Giustino A, Cagiano R, Carratù MR, Cassano T, Tattoli M, Cuomo V (1999): Prenatal exposure to low concentrations of carbon monoxide alters habituation and nonspatial working memory in rat offspring. Brain Res 844:201-205

Goodlett CR, Gilliam DM, Nichols J, West J (1989): Genetic influences on brain growth restriction induced by developmental exposure to alcohol. Neurotoxicology 10:321-324

Govoni S, Trabucchi M, Cagiano R, Cuomo V (1994): Alcohol and the brain: Setting the benefit/risk balance. Alc 11(3):241-246

Hannigan JH (1996): What research with animals is telling us about alcohol-related neurodevelopmental disorder. Pharmacol Biochem Behav 55(4):489-499

Hard E, Engel J, Larsson K, Liljequist S, Musi B (1985): Effects of maternal ethanol consumption on the offspring sensory-motor development, ultrasonic vocalization, audiogenic immobility reaction and brain monoamine synthesis. Pharmacol et toxicol 56:354-363

Insel TR, Hill JL, Mayor RB (1986): Rat pup ultrasonic isolation calls: Possible mediation by the benzodiazepine receptor complex. Pharmacol Biochem Behav 24:12631267

Kehoe P, Shoemaker W (1991): Opioid-dependent behaviors in infant rats: Effects of prenatal exposure to ethanol. Pharmacol Biochem Behav 39:389-394

Kim CK, Kalynchuk LE, Kornecook T (1997): Object-recognition and spatial learning and memory in rats prenatally exposed to ethanol. Behav Neurosci 111(5):985-995

Krahl SE, Berman RF, Hannigan JH (1999): Electrophysiology of hippocampal CA1 neurons after prenatal ethanol exposure. Alcohol 17(2):125-131

Laviola G, Renna G, Bignami G, Cuomo V (1988): Ontogenetic and pharmacological dissociation of various components of locomotor activity and habituation in the rat. Int J Develop Neurosci 6(5):431-438

Lester BM (1987): Developmental outcome prediction from acoustic cry analysis in term and preterm infants. Pediatrics 80:529-534
Maier SE, Chen WJ, West JR (1996): Prenatal binge-like alcohol exposure alters neurochemical profiles in fetal rat brain. Pharamacol Biochem Behav 55:521-529

McBride WJ, Chernet E, Lumeng L, Li TK (1993): Densities of dopamine D2 receptors are reduced in CNS regions of alcohol-preferring P rats. Alc 10:387-390

Melcer T, Gonzales D, Riley EP (1995): Locomotor activity and alcohol preference in alcohol-preferring and -nonpreferring rats following neonatal alcohol exposure. Neurotoxicol Teratol 17(1):41-48

Michelsson K, Sirvio P, Wasz-Hockert O (1977): Sound spectrographic cry analysis of infant with bacterial meningitis. Dev Med Child Neurol 19:309-315

Murphy JM, McBride WJ, Lumeng L, Li TK (1982): Regional brain levels of monoamines in alcohol-preferring and nonpreferring lines of rats. Pharmacol Biochem Behav 16:145-149

Ness JW, Franchina JJ (1990): Effects of prenatal alcohol exposure on rat pups' ability to elicit retrieval behavior from dams. Dev Psychobiol 23:85-99

Nulman I, O'Hayon B, Gladstone J, Koren G (1998): The effects of alcohol on the fetal brain. The fetal nervous system tragedy. In Slikker W JR, Chang LW (eds), Handbook of Developmental Neurotoxicology. San Diego, CA, Academic Press, pp 567-586

Paxinos G, Watson C (1986): The Rat Brain in Stereotaxic Coordinates. Sydney, Academic Press

Pozzi L, Trabace L, Invernizzi R, Samanin R (1995): Intranigral GR-113808, a selective $5-\mathrm{HT}_{4}$ receptor antagonist, attenuates morphine-stimulated dopamine release in the rat striatum. Brain Res 692:265-268

Riley EP, Lochry EA (1982): Genetic influences in the etiology of fetal alcohol syndrome. In Abel EL (ed), Fetal Alcohol Syndrome, 3, Animal Studies. Boca Raton, CRC Press, pp 113-130

Riley EP, Barron S, Melcer T, Gonzales D (1993): Alterations in activity following alcohol administration during the third trimester equivalent in $\mathrm{P}$ and NP rats. Alc Clin Exp Res 17:1240-1246

Sales G (1979): Strain differences in the ultrasonic behavior of rats (Rattus norvegicus). Amer Zool 19:513-527

Schulz PE, Fitzgibbons JC (1997): Different mechanism of expression for short- and long-term potentiation. J Neurophysiol 78:321-337

Shen RY, Hannigan JH, Kapatos G (1999): Prenatal ethanol reduces the activity of adult midbrain dopamine neurons. Alc Clin Exp Res 23(11):1801-1807

Spanagel R, Weiss F (1999): The dopamine hypothesis of reward: Past and current status. TINS 22:521-527

Stefanini E, Frau M, Garau MG, Fadda F, Gessa GL (1992): Alcohol-preferring rats have fewer dopamine D2 receptors in the limbic system. Alc Alcoholism 27: 127-130

Streissguth AP (1986) The behavioral teratology of alcohol: Performance, behavioral, and intellectual deficits in prenatally exposed children. In West JR (ed), Alcohol and Brain Development. New York, Oxford University Press, pp 3-44 